\title{
Echographie Doppler Arterielle Testiculaire Dans L'infertilite Masculine A Lome
}

\author{
Amadou A. \\ Sonhaye L.
}

Service de Radiologie et Imagerie du CHU Campus.

Douaguibe $B$.

Service de Gynécologie et Obtétrique du CHU Campus.

\section{Tchaou M.}

Service de Radiologie et Imagerie du CHU Sylvanus Olympio

\section{Agbangba $K A$. \\ Watara $G$. \\ Adjenou $V$. \\ N'dakena $K$.}

Service de Radiologie et Imagerie du CHU Campus.

\begin{abstract}
Objective: Determine the relationship between the testicular arterial resistance index (IR) and spermatogenesis. Material and method: prospective analytical study over a period of 12 months at CHU campus and National Institute of Hygiene of Lomé. The parameters studied were anomalies of sperm count, sperm volume, testis volume, and resistance index (RI). The study of the correlation between our variables was done using the Chi square test $(\chi 2)$. Results: The mean age of men was 36.26 years. Sperm volume was normal in $73 \%$ men and abnormal in $27 \%$ men. $65 \%$ had normal sperm counts, and $35 \%$ had abnormal sperm counts. The normal testicular volume was found in $42 \%$ men and $58 \%$ had an abnormal testicular volume (hypotrophic testis). 51\% men had the pathological RI while $49 \%$ had a normal RI. There was no statistically significant relationship between testicular volume and sperm count. Also, no statistically significant relationship between RI and testicular volume. But, there was a statistically significant correlation between RI and sperm count. No statistically significant difference between RI and sperm volume. Conclusion: RI can be used with sperm analysis and hormonal studies as part of the clinical evaluation of infertile men. An intratesticular RI greater than 0.6 is associated with decreased total
\end{abstract}


motile sperm.

Keywords: Testicles - Spermatogenesis - Doppler Ultrasound - Resistance Index

\section{Resume}

Objectif : Déterminer le rapport entre l'index de résistance (IR) artériel testiculaire et la spermatogenèse. Matériel et méthode : étude prospective analytique sur une période de 12 mois au CHU Campus et à l'Institut National d'Hygiène de Lomé dans cadre du bilan d'infertilité. Les paramètres étudiés étaient les anomalies de nombre des spermatozoïdes, le volume du sperme, le volume des testis et l'index de résistance (IR). L'étude de la corrélation entre nos variables a été faite à l'aide du test de Chi carré $(\chi 2)$. Résultats : Notre étude a concerné 100 sujets dont l'âge moyen était de 36,26 ans. Le volume de sperme était normal chez $73 \%$ des sujets et anormal chez $27 \%$ des sujets. $65 \%$ des sujets présentaient une numération normale des spermatozoïdes, contre $35 \%$ de numération pathologique de spermatozoïde. Le volume testiculaire normal était retrouvé chez $42 \%$ des sujets et $58 \%$ avaient un volume testiculaire anormal (testis hypotrophiques). Chez $51 \%$ des sujets, l'IR était pathologique alors que 49\% présentaient un IR normal. Il n'existait pas de lien statistiquement significatif entre le volume testiculaire et la numération des spermatozoïdes d'une part, entre le volume testiculaire et l'IR et d'autre part. Il a été retrouvé une corrélation statistiquement significative entre l'IR et la numération des spermatozoïdes. Mais il n'existait pas de relation statistiquement significative entre l'IR et le volume du sperme. Conclusion : L'IR peut être utilisé avec l'analyse du sperme et les études hormonales dans le cadre de l'évaluation clinique de l'homme infertile. Un IR intratesticulaire supérieur à 0,6 serait associé à une diminution du nombre de spermatozoïdes.

Mots clés : Testicules - Spermatogenése - Echographie Doppler - Index de résistance.

\section{Introduction}

L'infertilité est de plus en plus fréquente. Selon l'OMS, elle concerne environ $15 \%$ de couple, avec 30\% d'étiologie masculine. Au Togo, la stérilité conjugale représente $14 \%$ et la responsabilité masculine est engagée dans 47,06 \% des cas (Boukari et al, 1998), alors qu'en France, l'origine masculine serait de 20\% (Auger et al, 1995). L'infertilité masculine serait en rapport avec les anomalies de la spermatogenèse. Ainsi, le spermogramme demeure l'examen clé dans le bilan d'infertilité masculine. Mais l'imagerie occupe de 
plus en plus une place fondamentale. Elle est essentielle pour l'analyse morphologique de la glande testiculaire. Couplée au Doppler, elle permet une étude dynamique du système vasculaire veineux et artériel. Selon certaines études, un index de résistance (IR) anormal serait responsable des anomalies de la spermatogenèse. En Afrique noire, rares sont les études qui ont évalué la répercussion des anomalies vasculaires artérielles des testicules sur la spermatogenèse. Il nous a ainsi, paru opportun d'entreprendre ce travail dont l'objectif est de déterminer le rapport entre l'IR artériel testiculaire et la spermatogenèse afin de prédire l'influence des anomalies de la vascularisation artérielle testiculaire dans la spermatogenèse.

\section{Materiels Et Methode}

Notre étude a été prospective analytique et s'est déroulée dans le service de radiologie et imagerie médicale du Centre Hospitalier Universitaire (CHU) Campus sur une période de 12 mois. Elle a concerné les sujets ayant réalisé le spermogramme et l'échographie Doppler dans le cadre du bilan d'infertilité. Les paramètres étudiés étaient les anomalies de nombre des spermatozoïdes, le volume du sperme, le volume des testis et l'index de résistance (IR). Le nombre de spermatozoïdes était considéré normal s'il était supérieur à 15 millions par millilitre (Sharath Kumar, 2013). Le volume du sperme était considéré normal s'il était supérieur à $15 \mathrm{ml}$ Le volume testiculaire était considéré normal, si le volume moyen des deux testis était supérieur à $15 \mathrm{~cm} 3$ (le volume testiculaire est calculé par la formule : Longueur x Largeur x Epaisseur x 0,7) (Sharath Kumar, 2013). L'IR était considéré comme normal, si l'IR moyen des deux testis était inférieur à 0,62. (Attila, 1997) Les résultats du spermogramme ont été obtenu à partir du laboratoire biologique de l'Institu National d'Hygiène de Lomé Le matériel d'échographie utilisé était de marque Esaote MyLab muni de sondes multifréquences dont une sonde linéaire de 7,5 Mhz et comportant de modules Doppler couleur, Doppler pulsé et Doppler énergie. L'étude de la corrélation entre nos variables a été faite à l'aide du test de Chi carré $\left(\chi^{2}\right)$. Les résultats ont été considérés comme significatifs pour $\mathrm{p}<0,05$.

\section{Resultats}

Notre étude a concerné 100 sujets dont l'âge moyen était de 36,26 ans avec des extrêmes de 27 à 58 ans. Le volume de sperme était normal chez 73\% des sujets et anormal chez $27 \%$ des sujets. La numération normale des spermatozoïdes était retrouvée chez $65 \%$ des sujets, contre $35 \%$ de numération pathologique de spermatozoïde. Le volume testiculaire normal était retrouvé chez $42 \%$ des sujets et $58 \%$ avaient un volume testiculaire anormal (testis 
hypotrophiques). L'IR normal (figure 1) était retrouvé chez 49\% des sujets, alors que $51 \%$ des sujets présentaient un IR pathologique (figure 2).

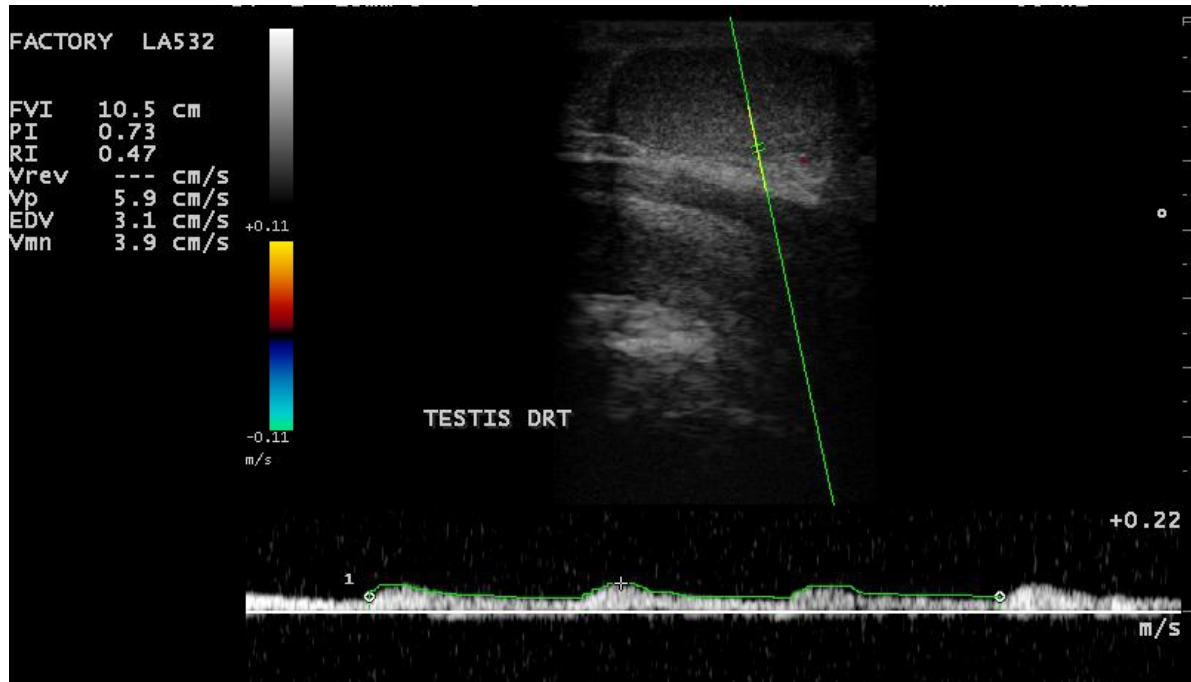

Figure 1 : Echographie Doppler pulsée testiculaire avec un IR normal $(0,47)$

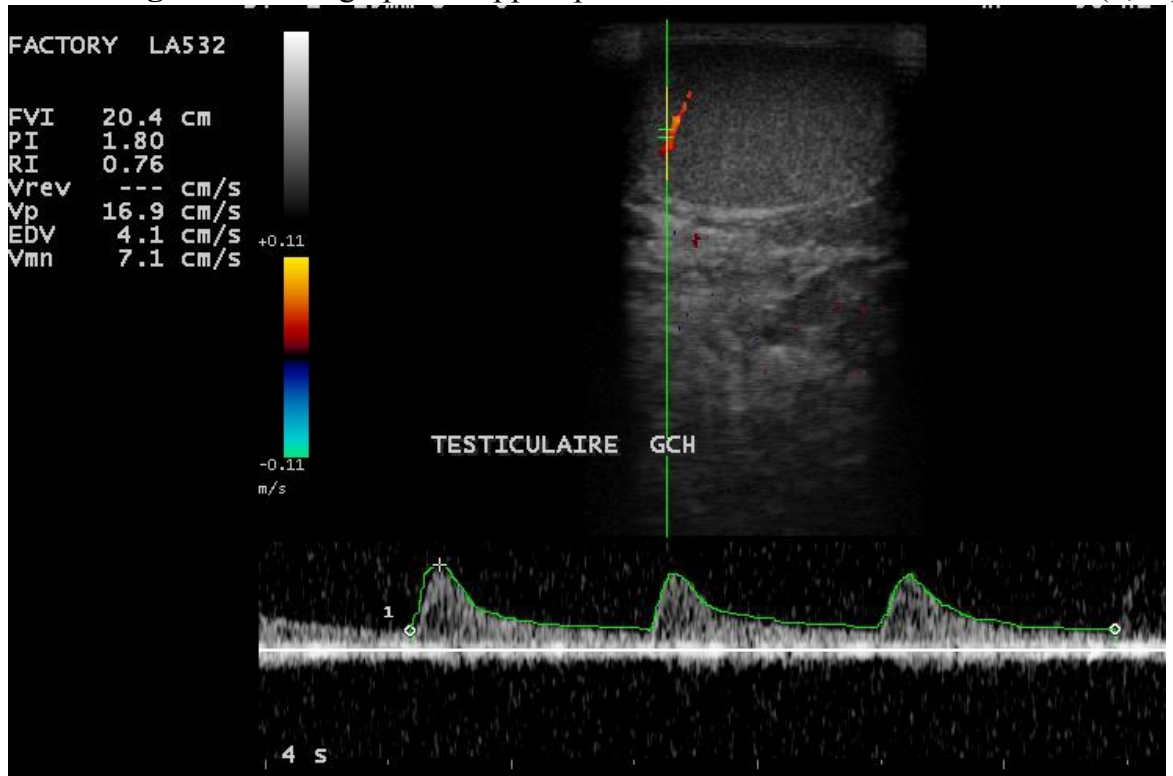

Figure 2 : Echographie Doppler pulsée testiculaire avec un IR pathologique $(0,76)$

L'hypotrophie testiculaire ainsi que le volume normal testiculaire prédominaient chez les sujets avec une numération de spermatozoïde anormale. Il n'existait pas de lien statistiquement significatif entre le volume testiculaire et la numération des spermatozoïdes (Tableau I).

Tableau I : Corrélation entre le volume testiculaire et la numération des spermatozoïdes

$<15 \mathrm{M} \quad>15 \mathrm{M} \quad$ Total




\begin{tabular}{|c|c|c|c|}
\hline Volume testis normal & 29 & 13 & 42 \\
\hline Volume testis anormal & 36 & 22 & 58 \\
\hline Total & 65 & 53 & 100 \\
\hline
\end{tabular}

L'IR pathologique était le plus retrouvé dans les cas de testis hypotrophiques, alors que l'IR normal était le plus retrouvé dans les cas de volume testiculaire normal, sans différence statistiquement significative (Tableau II).

Tableau II : Corrélation entre l'IR et le volume testiculaire

\begin{tabular}{|c|c|c|c|}
\hline & $<15$ & $>15$ & Total \\
\hline IR pathologique & 32 & 19 & 51 \\
\hline IR normal & 26 & 23 & 49 \\
\hline Total & 58 & 42 & 100 \\
\hline
\end{tabular}

Il n'existait pas de relation statistiquement significative entre l'IR et le volume testiculaire.

Les sujets avec une numération de spermatozoïde anormale présentaient majoritairement un IR pathologique, alors que l'IR normal était le plus retrouvé dans les cas de numération de spermatozoïde normale, avec une différence statistiquement significative (Tableau III).

Tableau III : Corrélation entre l'IR et de la numération des spermatozoïdes

\begin{tabular}{cccc}
\hline & $<10 \mathrm{M}$ & $>10$ & Total \\
\hline IR pathologique & $\mathbf{2 9}$ & $\mathbf{2 2}$ & $\mathbf{5 1}$ \\
IR normal & $\mathbf{0 6}$ & $\mathbf{4 3}$ & $\mathbf{4 9}$ \\
Total & $\mathbf{3 5}$ & $\mathbf{6 5}$ & $\mathbf{1 0 0}$ \\
\hline$\chi^{2}=21,86 \quad \mathrm{P}=0,000002 ;$ & Différence statistiquement significative &
\end{tabular}

Il existait une corrélation entre l'IR et la numération des spermatozoïdes. L'IR pathologique serait un facteur de risque d'anomalie de numération des spermatozoïdes. L'IR pathologique et l'IR normal étaient le plus retrouvé chez les sujets avec un volume anormal de sperme, sans différence statistiquement significative (Tableau IV).

Tableau IV : Corrélation entre l'IR et du volume de sperme

\begin{tabular}{cccc}
\hline & Volume Anormal & Volume Normal & Total \\
\hline IR pathologique & $\mathbf{1 2}$ & $\mathbf{3 9}$ & $\mathbf{5 1}$ \\
IR normal & $\mathbf{1 5}$ & $\mathbf{3 4}$ & $\mathbf{4 9}$ \\
Total & $\mathbf{2 7}$ & $\mathbf{7 3}$ & $\mathbf{1 0 0}$ \\
\hline$\chi^{2}=0,63 \quad \mathrm{P}=0,42 ;$ & Différence statistiquement non significative
\end{tabular}

Il n'existerait pas de relation statistiquement significative entre l'IR et le volume du sperme. Une anomalie de la vascularisation ne serait pas un facteur de la diminution du volume du sperme 


\section{Discussion}

L'échographie Doppler des testicules est un outil de diagnostic simple, important et reproductible, qui sert à recueillir les paramètres vélocimétriques des artères testiculaires, permettant ainsi l'évaluation de l'infertilité masculine (Attila et al, 1997 ; Biagiotti et al, 2002). Plusieurs études ont utilisé ce moyen d'exploration dans la prédiction de la perfusion testiculaire et de la spermatogenèse (Sharath Kumar, 2013 ; Al-Naffakh, 2012; Donkol, 2010 ; Zahalsky et al, 2001). L'échographie Doppler peut être utilisé chez tous les sujets et ceci quelque soit l'âge. Dans notre étude l'âge moyen des sujets était de 36,26 ans avec des extrêmes de 27 à 58 ans. La moyenne d'âge dans notre étude se rapproche de celle de Goullet et al (2000). Notre étude n'avait pas retrouvé de lien statistiquement significatif entre le volume des testis et le spermogramme d'une part, et entre l'IR et le volume testiculaire d'autre part. Nos résultats corroborent avec ceux de Polat et al (2015) qui révèlent une absence de lien statistiquement significatif entre le volume testiculaire et l'infertilité. Biagiotti et al (2002) n'avaient pas retrouvé de corrélation entre le nombre des spermatozoïdes et le volume testiculaire. Mais par contre, Sakamoto et al. (2008) avaient retrouvé une corrélation entre la fonction testiculaire et le volume testiculaire. Wielgos et al. (1999) ont trouvé un IR très élevée dans les cas d'hypotrophie testiculaire. Notre étude a retrouvé un lien statistiquement significatif entre l'IR et le spermogramme. Cette corrélation serait en rapport avec la numération et non avec le volume du sperme. En effet, le volume du sperme peut connaitre des fluctuations dues essentiellement au mode du recueil ou à une abstinence prolongée ou non.

Donc le volume du sperme ne serait pas lié à la vascularisation testiculaire. Al-Naffakh (2012) avaient notés une différence significative de l'IR entre les sujets fertiles et infertiles. Des études récentes (Attila et al, 1997 ; Biagiotti et al, 2002) ont montré que l'index de résistance (IR), mesuré à partir du débit systolique et diastolique maximal d'une artère testiculaire, est en corrélation avec la fonction testiculaire. Ces études (Attila et al, 1997 ; Biagiotti et al, 2002) ont démontré que l'IR serait un bon prédicteur de la fonction testiculaire et comme un moyen pour distinguer les différentes causes de dyspérmie. Selon Biagiotti et (2002), un IR plus élevé et une plus forte vitesse systolique (PSV) seraient corrélés avec l'azoospermie obstructive, et corrélée inversement avec le score du taux de production de spermatozoïdes.

Leur étude (Biagiotti et 2002) a constaté que seuls l'IR et le PSV étaient en corrélation avec le score de production de spermatozoïdes, alors que la FSH, le volume testiculaire et le volume diastolique (VED) ne l'étaient pas.

Des études de Pinggera et al. (2008) ont révélé qu'un IR supérieur à 0,6 est associé à une numération de spermatozoïdes pathologique. Ce qui est conforme aux résultats de notre étude. Hillelsohn et al (2013), en comparant les groupes avec un IR supérieur à 0,6 et un IR de 0,6 ou moins, ont trouvé 
une différence significative dans les spermatozoides total mobile $(\mathrm{P}<0,01)$. Cette constatation confirme l'hypothèse selon laquelle un IR supérieur à 0,6 est associé à une dyspérmie. Cette étude [14] a de plus, constaté que pour un IR supérieur à 0,6 , la sensibilité pour le spermatozoïde total mobile inférieur à $20 \times 106$ était de 57,14\%, et la spécificité était de 63,27\%, avec un rapport de vraisemblance de 1,56. Aussi, Un IR de 0,56 ou plus était-il également significativement associé à une mobilité totale plus faible $(\mathrm{P}=0,04)$.

L'explication de l'association du flux sanguin testiculaire et de la spermatogenèse n'a pas encore été complètement élucidée. Une étude menée par Foresta et al (1998) affirme que le nombre de branches artérielles irriguant les testicules chez les patients présentant une azoospermie est réduit ; il est inférieur à 04 branches alors chez les sujets fertiles, ce nombre dépasse 04 vaisseaux artériels par champ. Davantage de recherches sont nécessaires pour clarifier si la microcirculation testiculaire altérée telle que reflétée par un IR élevé est secondaire à un dysfonctionnement vasculaire systémique ou une conséquence de la diminution de la fonction testiculaire. Une corrélation de l'IR avec la biopsie testiculaire est nécessaire pour affirmer qu'une IR altérée identifie une dysfonction spermatogénique (Hillelsohn et al, 2013).

\section{Conclusion}

L'analyse Doppler spectrale de l'homme infertile a plusieurs applications cliniques actuelles et potentielles, notamment pour l'évaluation de la fonction testiculaire chez l'homme infertile. De notre étude, il ressort qu'un IR intratesticulaire supérieur à 0,6 serait associé à une diminution de la spermatogenèse, contrairement à la diminution du volume testiculaire. Ainsi donc l'échographie Doppler pulsée serait nécessaire dans le bilan de l'infertilité masculine dans le but d'évaluer la vascularisation artérielle testiculaire.

\section{Déclaration D'intérêts} cet article.

Les auteurs déclarent ne pas avoir de conflits d'intérêts en relation avec

\section{References:}

1. Boukari BS Boukari BS, Adjahoto EO, Anoukoum T, Folligan K, Djobo BM, Napo Koura GA. Aspect clinique de la stérilité masculine au CHU Tokoin de Lomé. Andrologie 1998 ; 8 : 302-6.

2. Auger J, Kustmann JM, Czyglik F, Jouannet P. Decline in semen quality among fertile man in Paris during the past years. New Engl. J.Med, 1995; 332: 281-5. 
3. Sharath Kumar C. et al. Assessment of Testicular Volume in Correlation with Spermiogram of Infertile Males in South India. Advanced Studies in Biology. 2013; 5: 327 - 35

4. Atilla MK, Sargin H, Yilmaz Y, Odabas O, Keskin A, Aydin S. Undescended testes in adults: clinical significance of resistive index values of the testicular artery measured by Doppler ultrasound as a predictor of testicular histology. J Urol 1997; 158: 841-3

5. Biagiotti G, Cavallini G, Modenini F, Vitali G, Gianaroli L. Spermatogenesis and spectral echo-colour Doppler traces from the main testicular artery. BJU Int 2002; 90 : 903-8

6. Al-Naffakh H. Testicular Doppler resistive index parameter as predictor test for male infertility. Kufa Med. Journal 2012; 15: 293-7.

7. Donkol RH. Imaging in male-factor obstructive infertility. World J Radiol 2010; 2:172-9.

8. Zahalsky M, Nagler HM. Ultrasound and infertility: diagnostic and therapeutic uses. Curr Urol Rep 2001; 2:437- 42.

9. Goullet E, Rigot J, Blois N. Intérêt de l'échographie scrotale systématique dans la prise en charge de l'homme infertile. Etude prospective de 609 cas. Urologie $2000 ; 10: 78-82$.

10. Polat H, Sarica MA, Bulut HT, Yucel MO, Gok A, Cift A, Kalyenci B. The relationship between mean platelet volume and testicular blood flow and fertility. Int J Clin Exp Med 2015; 8 :11554 - 8

11. Sakamoto H, Yajima T, Nagata M, Okumura T, Suzuki K, Ogawa Y. Relationship between testicular size by ultrasonography and testicular function: Measurement of testicular length, width, and depth in patients with infertility. International Journal of Urology 2008; 15 : 529-33

12. Wielgos M, Frącki S, Bablok L, Rokicki T, Marianowski L. Testicular artery Doppler flow measurements in patients with the Klinefelter syndrome. Med Sci Monit, 1999; 5 1197- 9

13. Pinggera GM, Mitterberger M, BartschG, Strasser H, Gradl J, Aigner F, Pallwein L, Frauscher F. Assessment of the intratesticular resistive index by colour Doppler ultrasonography measurements as a predictor of Spermatogenesis. Journal compilation 2008; BJU international $101 ; 722-6$

14. Hillelsohn JH, Chuang KW, Goldenberg E, Gilbert BR. Spectral Doppler Sonography: A Noninvasive Method for Predicting Dyspermia. J Ultrasound Med. 2013 ; 32: 1427-32

15. Foresta C, Garolla A, Bettella A, Ferlin A, Rossato M, Candiani F. Doppler ultra sound of the testis in azoospermic subjects as parameter of testicular function. Human reproduction 1998; 13: 3090- 3 